\title{
Title: Functional impact of genomic complexity on the transcriptome of Multiple Myeloma
}

Bachisio Ziccheddu ${ }^{1,10 \#}$, Matteo C. Da Vià, ${ }^{2,3}$, Marta Lionetti ${ }^{2,3}$, Akihiro Maeda ${ }^{3}$, Silvia Morlupi ${ }^{3}$, Matteo Dugo ${ }^{4}$, Katia Todoerti ${ }^{2,3}$, Stefania Oliva ${ }^{1}$, Mattia D'Agostino ${ }^{1}$, Paolo Corradini ${ }^{2,3}$, Ola Landgren $^{6,7,10}$, Francesco Iorio ${ }^{8,9}$, Loredana Pettine ${ }^{2}$, Alessandra Pompa ${ }^{2}$, Martina Manzoni ${ }^{2,3}$, Luca Baldini $^{2,3}$, Antonino Neri ${ }^{2,3}$, Francesco Maura ${ }^{6,7,10, \$}$, and Niccolò Bolli ${ }^{2,3, \$}$

${ }^{1}$ Department of Molecular Biotechnologies and Health Sciences, University of Turin, Turin, Italy.

${ }^{2}$ Hematology Unit, Fondazione IRCCS Ca' Granda Ospedale Maggiore Policlinico, Milan, Italy.

${ }^{3}$ Department of Oncology and Hemato-Oncology, University of Milan, Milan, Italy.

${ }^{4}$ Platform of Integrated Biology, Department of Applied Research and Technology Development, Fondazione IRCCS

Istituto Nazionale dei Tumori, Milan, Italy.

${ }^{5}$ Department of Hematology, Fondazione IRCCS Istituto Nazionale dei Tumori, Milan, Italy.

${ }^{6}$ Myeloma Service, Department of Medicine, Memorial Sloan Kettering Cancer Center, New York, New York, USA.

${ }^{7}$ Department of Medicine, Weill Cornell Medical College, New York, NY, USA.

${ }^{8}$ Centre for Computational Biology, Human Technopole, Milan, Italy.

${ }^{9}$ Cancer, Ageing and Somatic Mutation Programme, Wellcome Sanger Institute, Cambridge, UK.

${ }^{10}$ Myeloma Program, Sylvester Comprehensive Cancer Center, University of Miami, Miami, FL, USA.

"These authors contributed equally

${ }^{\$}$ These authors jointly supervised the work

Running title: Myeloma genomic complexity transcriptional impact

\section{Correspondence to:}

Francesco Maura, MD,

Myeloma Program, Sylvester Comprehensive Cancer Center, University of Miami, 1120 NW 14th Street, Clinical Research Building

Miami, FL 33136

Phone: (305) 2437687

E-mail: fxm557@med.miami.edu

\section{Niccolò Bolli}

Hematology Unit, Fondazione IRCCS Ca' Granda Ospedale Maggiore Policlinico, Milan, Italy. Department of Oncology and Hemato-Oncology, University of Milan, Milan, Italy.

Via Francesco Sforza, 35. -20122- Milan, Italy.

Phone: +390255033337

Email: niccolo.bolli@unimi.it

\section{Conflicts of interest}

All authors declare that the research was conducted in the absence of any commercial or financial relationships that could be construed as a potential conflict of interest. NB received honoraria from Celgene, Amgen and Janssen. 


\section{Translational relevance:}

In this paper we studied the close interactions between the genomic architecture of Multiple Myeloma and its functional impact on the transcriptome. This comprehensive analysis highlighted how mostly copy number changes and chromosomal rearrangements impacted gene expression. However, we showed the great importance that mutations play in the setting of bi-allelic inactivation of tumor suppressor genes. Moreover, a widespread geno-transcriptomic analysis was able to infer the differential gene expression of specific druggable pathways and to identify biomarkers of sensitivity to novel treatment in Multiple Myeloma, suggesting this approach could drive personalized treatment decisions. 


\begin{abstract}
Purpose: Multiple Myeloma (MM) is a biologically heterogenous plasma-cell disorder. In this study we aimed at dissecting the functional impact on transcriptome of gene mutations, copynumber abnormalities (CNAs), and chromosomal rearrangements (CRs). Moreover, we applied a geno-transcriptomic approach to identify specific biomarkers for personalized treatments.
\end{abstract}

Methods: We analyzed 514 newly diagnosed patients from the IA12 release of the CoMMpass study, accounting for mutations in MM driver genes, structural variants, copy-number segments and raw-transcript counts. We performed an in-silico drug sensitivity screen (DSS), interrogating the DepMap dataset after anchoring cell lines to primary tumor samples using the Celligner algorithm.

Results: Immunoglobulin translocations, hyperdiploidy and Chr(1q)gain/amps were associated with the highest number of deregulated genes. Other CNAs and specific gene mutations had a lower but very distinct impact affecting specific pathways. Many recurrent genes showed a hotspot(HS)specific effect. The clinical relevance of double-hit MM found strong biological bases in our analysis. Bi-allelic deletions of tumor suppressors and chr(1q)-amplifications showed the greatest impact on gene expression, deregulating pathways related to cell-cycle, proliferation and expression of immunotherapy targets. Moreover, our in-silico DSS showed that not only $\mathrm{t}(11 ; 14)$ but also chr(1q)gain/amps and CYLD inactivation predicted differential expression of transcripts of the $B C L 2$-axis and response to venetoclax.

Conclusions: The MM genomic architecture and transcriptome have a strict connection, led by CNAs and CRs. Gene mutations impacted especially with HS-mutations of oncogenes and bi-allelic tumor suppressor gene inactivation. Finally, a comprehensive geno-transcriptomic analysis allows the identification of specific deregulated pathways and candidate biomarkers for personalized treatments in MM.

Keywords: Multiple myeloma, personalized medicine, genomics, transcriptomics, BCL2 inhibitors. 


\section{INTRODUCTION}

Multiple Myeloma (MM) is a plasma cell neoplasm driven by recurrent trisomies -hyperdiploid MM (HDMM)- or by immunoglobulin gene ( $\operatorname{IgH})$ locus rearrangements promoting overexpression of recurrent oncogenes. Classical PCR and FISH studies, and more recently next-generation sequencing (NGS) studies have highlighted a vast array of additional mutations, copy-number abnormalities (CNAs) and structural variants (SVs) which are thought to play a role in disease evolution and may be acquired in pre-clinical phases or at relapse after treatment (1-9).

Such abnormalities are predicted to influence the biological and clinical behavior of the tumor and yet, despite a clear driver role, only few carry prognostic value (2,10-12). Additionally, as the genomic makeup of MM is being comprehensively elucidated by NGS studies, classification efforts seem to have gained very little by the additional knowledge brought by NGS and initiating karyotypic events are still the mainstay of MM classification. Indeed, most additional abnormalities discovered by NGS seem to be randomly distributed with few notable exceptions $(13,14)$ and their discovery has not led to the characterization of additional MM categories so far. If not relevant from the point of view of a genomic classification then, a question is what their biological role really is, and whether they can serve as markers of specific transcriptional profiles.

Classically, gene-expression profiling arrays have shown how transcriptome profiling recapitulates the main karyotypic lesions in MM (15-17). Few studies on RNAseq in MM have been published (7,14,18-21). Most of them have reported that many DNA mutations are actually not expressed, and how the transcriptomic profile has very few genomic correlates aside from hyperdiploidy and IGH translocations. Laganà et al have shown that RNAseq from the public CoMMpass database can be mined through a network approach to identify MM groups where annotation of genomic and clinical features highlights some commonalities (20). However, a systematic and comprehensive assessment of the functional impact of mutations, CNAs and SV at the transcriptomic level is still lacking. This would have implications for disease pathogenesis and would allow prioritization of research on genomic abnormalities that have specific consequences on expression. Ideally, those would be the ones with the highest value as prognostic markers, biomarkers of drug response, and/or candidate drug targets. Indeed, while for example response to venetoclax is best predicted by assessing the expression levels of $B C L 2, B C L 2 L 1$ and MCL1 (22), this is unlikely to become routine clinical practice. DNA abnormalities that correlate with expression of target genes are much more easily amenable to clinical-grade diagnostics through NGS techniques (23-25).

In this paper, we mined genomic and transcriptomic data from 514 cases from the CoMMpass dataset to dissect transcriptional effects of the most recurrent genetic abnormalities. In particular, starting from the comprehensive DNA exome-wide sequencing information, we performed a systematic and unbiased assessment of differentially expressed transcripts correlated to each recurrent gene mutation, CNA and SV. From here, we identified genetic predictors of specific differential gene expression levels to get insights into the functional relevance of recurrent genomic alterations and suggest therapeutic vulnerabilities.

\section{MATERIALS AND METHODS}

\section{Data}

We interrogated 514 newly diagnosed patients enrolled in the CoMMpass study (IA12 release). The CoMMpass data were generated as part of the Multiple Myeloma Research Foundation Personalize Medicine Initiative (https://themmrf.org). We focused on mutations in MM driver genes, structural variants, copy number segments and raw transcript counts. The CoMMpass trial is a prospective observational clinical trial (NCT01454297) that comprises whole genomic and transcriptomic data derived from newly diagnosed MM patients. The tumor samples were collected from several centers across the United States, Canada and Europe. Ethics committees or institutional review boards at the study sites approved the study. All the patients signed a written informed consent prior 
to enrollment. The study was conducted under the statement of the Helsinki declaration. MM samples were collected at diagnosis at enrollment sites and then shipped to the Translational Genomics Research Institute (TGen) in Phoenix, Arizona to be processed and sequenced. Due to the lack of transcriptomic data from normal plasma cells in the CoMMpass dataset, all the statistical analyses were performed comparing genetic subsets of MM samples.

\section{Gene expression analysis}

RNAseq data was processed using the voom/limma pipeline $(26,27)$. Firstly, the dataset of rawcounts was filtered to remove genes with $<10$ reads in $>95 \%$ of samples. Then, we performed the trimmed mean of M-values (TMM) normalization to estimate a scale factor used to decrease technical bias between samples, resulting from differences in library size (28). Finally, we applied the voom transformation to convert the raw counts in $\log 2$-counts per million $(\log 2-\mathrm{CPM})$ and calculated the respective observation-level weights to be used in differential expression analysis.

To assess the amount of differentially expressed transcripts a linear model was fitted to the expression data for each genomic feature, detecting the genes significantly associated with at least one genetic abnormality. This model evaluates the expression of each gene in each patient using a design matrix composed of information on genetic anomalies for each patient. From this, we obtained the coefficients that measure the gene expression change associated with the presence of each genetic anomaly. Then, to determine the association between gene expression and genomic alterations we used the F statistic obtained by the $1 \mathrm{mFit}$ function of the $\mathrm{R}$ limma package. Finally, we applied the Benjamini-Hochberg correction for multiple testing. To evaluate the accuracy of the applied model, we calculated a random model in which all the values of each covariate (genetic anomaly) were randomly permuted, breaking all the correlations between the gene expression level and genotype (29). This model allowed to correct the subsequent differential expression analysis for the main karyotypic subtypes and segmental CNAs [hyperdiploidy, $\mathrm{t}(4 ; 14), \mathrm{t}(11 ; 14), \mathrm{t}(14 ; 16)$, $\mathrm{t}(14 ; 20)$ and $\mathrm{chr}(1 \mathrm{q} 21)$ gains/amplifications (amp)].

Moreover, this approach allows us to identify significant gene associations by calculating the coefficient of determination (R2), which tells us how well the model fits the data, but also tells us the portion of variance of the dependent variable (expression of gene in a patient) predicted by the independent variable (presence of genetic anomaly). This allows us to measure the variance explained by the presence-absence of a given genetic alteration and to evaluate how much this alteration is associated with changes in expression of the candidate gene.

\section{Hotspot and non-hotspot mutations}

Mutational hotspots (HS) are amino acid positions in a protein-coding gene mutated more frequently than would be expected in the absence of selection (30), therefore conferring a fitness advantage. For this analysis, we selected 4 genes from a list of 53 mutations on known myeloma driver genes (13): KRAS, NRAS, IRF4 and BRAF. Then, we defined as HS the non-synonymous mutations as follows: G12, G13 and Q61 were selected as HS for KRAS and NRAS. K123 for IRF4 and V600 and D594 codons for BRAF.

\section{Identification of genes with a cumulative effect}

Cumulative transcriptomic effect of genes was analyzed in regions of chr(1q21) amplifications (>3 copies) or bi-allelic inactivation of tumor suppressors (mutation of one copy and deletion of the other). To explore the functional significance of these events, we selected genes with a cumulative effect, i.e. genes significantly deregulated in bi-allelic vs wild-type [or amp vs wild-type for chr(1q21)] and/or bi-allelic vs. mono-allelic statuses [or amp vs gain for chr(1q21)]. To perform this analysis, first we selected, for each analysis, the significant genes from the differential expression analysis contrasts, then for each selected gene we calculated with a linear regression 
their cumulative effect. Finally, we restricted analysis to the significant genes $(P$ value $<0.05)$ deregulated in bi-allelic (or amp) vs wild-type and/or bi-allelic (or amp) vs mono-allelic (or gain).

\section{Gene Ontology analysis}

The Gene Ontology (GO) enrichment analysis was performed using ClusterProfiler R package to search for a biological interpretation of transcriptome deregulation. This algorithm implements methods to analyze and visualize functional profiles of genomic coordinates, genes and gene clusters (31). The $P$ values were corrected for multiple testing by using the Benjamini-Hochberg false discovery rate (FDR) method. In the HS analysis, we performed the pathway analysis with the genes from differential expression analysis contrasts (HS vs wild-type, HS vs nonHS and nonHS vs wild-type); whereas in the bi-allelic deletions and chr(1q21)gain/amp analysis, we executed the GO analysis with the cumulative effect genes. Gene set enrichment analysis was performed by the $\mathrm{R}$ package "fgsea" using the " $t$ " statistic as ranking factor. The cancer hallmark list was inferred with a significant adjusted $P$ value of $<0.05$ (32).

\section{In-silico analysis of drug sensitivity}

To assess our findings, we performed an in-silico analysis using the DepMap database and Celligner algorithm (detailed methods in the Supplementary_Data1) (33-35).

\section{Statistical analysis}

The association tests have been performed with two-sided Wilcoxon rank sum test. The $P$ values were adjusted using the Benjamini-Hochberg FDR method (FDR $<0.05)$. All analyses were performed in $\mathrm{R}$, the language and environment for statistical computing (R Core Team, 2020).

\section{RESULTS}

\section{Genome-wide transcriptome patterns associated with MM driver aberrations}

To have an overview of the driver genomic lesions underlying global transcriptome deregulation in MM, we first performed an unsupervised principal component analysis (PCA). Cases segregated mostly based on their HD vs. IGH translocation status (Fig. 1A), in agreement with historical gene expression profiling data $(15,17,36,37)$. However, recent genomic efforts have greatly expanded the repertoire of driver genomic lesions in MM, including copy-number abnormalities and gene mutations. Therefore, we asked what the independent contribution of each to the derangement of the transcriptome could be. To this end, we implemented a linear regression model that assesses which transcripts are specifically associated to distinct genomic features (29). We plotted, for each driver lesion, the number of up- or down-regulated transcripts and their fold-change (Fig. 1B). IGH translocations and hyperdiploidy were associated overall with the most substantial differential gene expression patterns observed, along with amplifications and gains in chr(1q21). Other CNAs had fewer transcriptomic correlates, followed by specific gene mutations. Within the former group, CYLD deletions showed the highest number of differentially expressed transcripts compared with normal $C Y L D C N$ status. Mutations of the 53 known driver genes in MM (13) were characterized by less consistent transcriptomic patterns. However, interesting correlations emerged from this analysis. DIS3 and NRAS mutations were the two lesions whose occurrence was associated with the largest transcriptional modulation with respect to germline configuration. A high number of differentially expressed lncRNAs was observed in DIS3-mutated versus wild-type patient samples in particular, confirming DIS3 may mostly regulate expression at the post-transcriptional level (Supplementary_Data2) (38). As expected, GO analysis for NRAS and KRAS mutations showed differentially expression of genes pertaining to the MAPK pathway (Supplementary Fig. S1A-B). Among less frequent mutations, TGDS and RBI in 13q and the $M Y C$ transcriptional regulators IRF4 and $M A X$ displayed correlation with the transcriptome. Conversely, most of the remaining gene mutations were not associated with any differentially expressed transcript. 
IGH translocations exert their transforming activity by overexpressing target oncogenes. Indeed, modelling predicted vs observed expression of $C C N D 1$ showed that the $\mathrm{t}(11 ; 14)$ accounted for most of the observed inter-patient heterogeneity in CCND1 transcript levels (Fig. 1C, left), as was the case for NSD2 and to a lesser extent- FGFR3 expression in $\mathrm{t}(4 ; 14), M A F$ in $\mathrm{t}(14 ; 16)$ and $M A F B$ in $\mathrm{t}(14 ; 20)$ (Supplementary Fig. S2A-D). In the case of CNAs, the picture was not as clear-cut: while low $C Y L D$ expression levels were mostly explained by locus deletions (Fig. 1C, right), not all cases of $\operatorname{chr}(17 \mathrm{p} 13)$ or TRAF3 gene deletions resulted in down-regulation of TP53 or TRAF3, respectively (Supplementary Fig. S2E-F). In the case of chr(1q21)gain/amp, heterogeneity of expression levels of the key target genes $C K S 1 B$ and $M C L 1$ could be attributed to the allelic status of the locus only to an extent (Supplementary Fig. S2G-H).

Therefore, structural, numeric variants and few mutations in driver genes were associated with most of the transcriptomic heterogeneity between MM cases. For canonical IGH translocations this is likely mediated by overexpression of the target oncogene. The transcriptomic inter-patient variability observed in the case of CNAs is likely associated with the altered expression of more than one target gene and can be partially explained by the clonality of the event.

\section{Impact of driver recurrent gene mutations on gene expression regulation}

Despite having a smaller correlation with the transcriptome, recurrent gene mutations have a clear driver role. Many genes are mutated in a hotspot pattern, and specific effects of HS vs non-HS variants as compared to their wild-type (WT) status are unknown. We therefore sought to analyze genotype-phenotype correlations for 4 of the main mutated genes with known HS: KRAS, NRAS, $B R A F$ and IRF4. The distribution of HS and non-HS mutation within the main cytogenetic categories was neutral except for kinase-dead BRAF mutations at codon D594, mostly occurring in $\mathrm{t}(14 ; 16)$ cases (Supplementary Fig. S3). As a first step, we interrogated the Catalogue of Somatic Mutations in Cancer (COSMIC) to interrogate the codon usage of these gene mutations in CoMMpass as compared to different solid cancers types (colon, lung adenocarcinoma and skin melanoma) and other hematological malignancies. Interestingly, MM showed a specific mutational spectrum for some of these genes. KRAS in particular was mostly mutated in the Q61 codon in MM, while in solid cancers, diffuse-large B cell lymphomas, chronic lymphocytic leukemia (CLL) and acute myeloid leukemia (AML) showed mostly G12 and G13 mutations (Fig. 2A and Supplementary Fig. S4A). NRAS was mainly mutated at the Q61 codon. A similar picture was also observed for solid cancers. However, in this latter setting, G12 and G13 residues mutations were also enriched in comparison to MM (Fig. 2B). Interestingly, other hematological malignancies showed a predominance of NRAS G12 and G13 mutations, and only CLL showed a mutational pattern similar to MM (Supplementary Fig. S4B). The IRF4 K123 HS was specific for MM. Nevertheless, we identified other IRF4 mutated HS in B and in T cell neoplasms, all within the DNA binding domain. In solid cancers, where IRF4 is not usually expressed, we did not identify any clustered mutational locus (Fig. 2C and Supplementary Fig. S4C). Finally, while BRAF mutations were mostly clustered on the V600, MM and other hematological malignancies showed a higher incidence of the "kinase-dead" D594 mutations as compared to solid tumors (Fig. 2D and Supplementary Fig. S4D). Notable exceptions were hairy cell leukemia and Langherans' Histiocytosis, where only the V600 residue resulted mutated (Supplementary Fig. S4D).

Next, we asked whether HS and non-HS mutations would carry different functional relevance as assessed by transcriptomic analysis: for $K R A S$ and $N R A S$, we found transcriptomic correlates for HS gene mutations only. Interestingly, even if the two genes fuel the same proliferative pathway (Supplementary Fig. S1), NRAS HS mutations were associated with differential expression of more than 2000 genes, as compared to 80 for KRAS HS mutations (Fig. 2AI, Fig. 2BI and Supplementary Fig. S5A-B). In the case of IRF4, again HS mutations correlated with the highest number of differentially expressed transcripts, while non-HS mutations were characterized by a less substantial and partly-overlapping transcriptional profile (Fig. 2CI and Supplementary Fig. S5C). Interestingly, 
for BRAF mutations, the D594 codon was correlated with the highest level of differential gene expression, showing some overlap with mutations in the canonical codon V600. In particular, the kinase-dead mutations at D594 were associated with the differential expression of 183 genes, three times more than the V600 ones. This may be partly explained by the specific occurrence of D594 mutations in $M A F$-translocated MM subgroup (4/7 cases) as previously described (14). Overall, non-HS mutations contributed very little in BRAF (Fig. 2DI and Supplementary Fig. S5D). Therefore, transcriptomic correlates seem to correspond mostly to HS mutations, and the pattern of HS usage seem to be specific for MM as compared to other solid and hematological cancers.

\section{Functional impact of bi-allelic events affecting tumor suppressor genes in multiple myeloma}

Bi-allelic events carry an increasingly recognized prognostic effect in MM. In particular, double-hit events occurring in tumor suppressor genes have been described to have a functional impact on cell fitness and survival and to represent the reservoir for disease relapse and drug resistance in more advanced stages $(10-12,39)$. Therefore, we sought to explore the transcriptomic consequences of biallelic events and how these are different from mono-allelic ones in key tumor suppressors: FAM46C, DIS3, RB1, TGDS, TRAF3, CYLD and TP53.

To this end, we looked at genes with a cumulative effect, i.e. genes significantly deregulated in biallelic vs WT and/or bi-allelic vs. mono-allelic statuses. Overall, among MM patients' samples the monoallelic status was associated with little transcriptomic change as compared to WT, while biallelic events showed more profound changes (Supplementary Fig. S6, Supplementary Table S1 and Supplementary_Data3). Then, to better explain the putative functional impact of gene differential expression, we performed GO analysis on genes with a cumulative effect. As a common feature, we found a generalized upregulation of genes involved in cell-cycle regulation and cell proliferation. Interestingly bi-allelic inactivations of TP53, TGDS and RB1 upregulated similar cyclins (CCND2, $C C N A 2, C C N E 2)$ and spindle checkpoints genes (E2F1 and $M C M s)$. Furthermore, they shared also upregulation of aurora kinase-A (AURKA), cyclin-dependent kinases (CDK1 and CDK2) and Polokinase-1 (PLK1), genes for which targeted treatments are being trialed (Supplementary Fig. S7). These results were confirmed by a gene set enrichment analysis (GSEA). Indeed, we observed that cases with inactivation of TP53, TGDS, RB1_or FAM46C shared many cancer hallmarks gene sets, all related to cell-cycle regulation and MYC (Supplementary Fig. S8).

However, each gene aberration also showed a specific transcriptomic profile associated with biallelic inactivation. $C Y L D$ double-hit events showed as expected upregulation of downstream effectors of the NF-kB pathway such as BIRC3, TRAF4, NFKB2, IKBKE, RELB and TNFAIP3 (Fig. 3A, upper panel, and Supplementary Fig. S8). Moreover, we also detected an upregulation of genes involved in the interaction with microenvironment as ICAM1, EDN1 and FGF2 related to angiogenesis enhancement (40), or MIRI46A implicated in the communication with mesenchymal stromal cells. Interestingly, $C Y L D$ inactivation correlated with a significant overexpression of $B C L 2$, with a cumulative effect for bi-allelic events (Fig. 3A, lower panel). In the case of TP53 double-hits, we noted an upregulation of cell-cycle genes as well as $P H F 19$, a recently discovered poor prognostic marker in MM (Fig. 3B) (41). Therefore, we further explored this finding applying GSEA to dissect possible interactions between TP53 inactivation and PHF19 overexpression. Interestingly, the two abnormalities showed an overlap in upregulation of cell-cycle related and MYC controlled pathways (Supplementary Fig. S8), suggesting a convergence of mechanisms underlying poor prognosis in MM. Furthermore, TP53 double-hits were associated with downregulation of SLAMF7, an immunotherapeutic target in MM (Fig. 3B, lower panel). Interestingly, this was only observed in double-hit events and not when TP53 was mutated or deleted only. FAM46C double-hit events were characterized by an upregulation of $C D 38$ and a concomitant low expression of CD55 and CD59, recently associated with Daratumumab resistance (Supplementary Fig. S7A) (42). Moreover, we found an upregulation of CD44, a gene involved in MM cell homing and lenalidomide resistance (43). Then, we analyzed the impact of $\operatorname{chr}(13 q)$ 
deletions and mutations of genes located within this region (Supplementary Fig. S7B-D). TGDS and $R B 1$ bi-allelic events deregulated mostly genes involved in cell-cycle and cell proliferation. Doublehit events of $T G D S$ were predominantly associated with gene upregulation, while patients with $R B 1$ bi-allelic inactivations were characterized by differentially expressed genes in both directions compared with samples with normal/mono-allelic status of the gene. DIS3 alterations correlated with increased expression of lncRNAs regulatory genes (Supplementary Fig. S7B-D) as expected given its function (38). TRAF3 inactivation was associated with NF-kB pathway activation and downregulation of signaling related to mRNA metabolic processes and protein catabolism (Supplementary Fig. S7E). Overall, these findings may help to explain the putative biological and prognostic impact of bi-allelic events in MM.

\section{Chromosome 1q gain/amplifications impacted transcriptome}

Another recurrent CNA in MM affects chr(1q21), where gains (3 copies) are thought to be early events and amplifications (4 or more copies) late events with a negative prognostic impact $(11,13)$. As for chromosomal deletions, also in this case we observed several genes characterized by a progressively increased change in expression in diploid vs gain vs amplifications of chr(1q21). While an intermediate differential expression was observed in the gain group compared with the disomic one, greater expression z-scores of these altered transcripts characterized the amplified cohort (Fig. 4A). As this was the single CNA with the largest number of transcriptomic correlates (Fig. 1B), we asked whether alteration of expression only impacted genes in the duplicated region or was genome-wide. Taking into account the number of differential expressed genes in each chromosome corrected for the chromosomal length, we observed how small chromosomes (i.e. 21 or 22) contributed proportionally as much as chromosome 1 to transcriptomic changes between normal disomic configuration and 1q extra-copies. This suggests that chr(1q21)gain/amp may have profound implications on the transcriptome of the cell which are genome-wide (Fig. 4B). Subsequently, we applied a gene ontology analysis on the genes with 1q CN-associated differential expression. Proliferation-related pathways, i.e. cell-cycle and spindle checkpoint genes were significantly upregulated (Fig. 4C). Chr(1q21)amp was associated with downregulation of CCND1 and upregulation of CCND2 along with other oncogenes (AURKA, AURKB and PLK1) confirming the view that MM is a disease of deregulated cyclins (Supplementary Fig. S9A-B) (44). Given the known association between chr(1q21)gain/amp and $\mathrm{t}(4 ; 14)$, we asked whether chr(1q21)amp effect on $C C N D 1$ and $C C N D 2$ could be mediated by $\mathrm{t}(4 ; 14)$. Firstly, we noted that despite the presence of 1102 shared differentially expressed genes between the two genetic abnormalities, chr(1q21)gain/amp was independently associated with 1191 differentially expressed genes and $\mathrm{t}(4 ; 14)$ with 3841 (Supplementary Fig. S10A). Of note, while CCND1 and CCND2 were differentially expressed by both abnormalities, cases of chr(1q21)gain/amp without $t(4 ; 14)$ also showed CCND2 upregulation (Supplementary Fig. S10B), confirming this is a convergent independent downstream effect of the two abnormalities and not a confounding effect of their association. Other than this, a GO analysis of differentially expressed genes specific to each genetic abnormality did not show shared deregulated pathways (Supplementary Fig. S10C-D). Finally, several genes with a possible therapeutic impact were differentially expressed. In particular, PDL1 was downregulated, while the immunotherapy targets SLAMF7 and GPRC5D were significantly overexpressed (Fig. 4D, Supplementary Fig. S9C). Of note, the chromosome 1q amplification was also associated with a significant upregulation of MCL1 (Fig. 4E), whose overexpression is associated with a lower response to BCL2 inhibition. Altogether, these data confirmed the high impact that chromosome 1q alterations have on the transcriptome and of great importance are the highly significant expression changes on genes with possible therapeutical implication for new generation immunotherapies (SLAMF7 and GPRC5D) or targeted treatments (MCL1). 


\section{Structural chromosomal alterations as possible predictors of response to BCL2 inhibitors}

Venetoclax is becoming a commonly used therapeutic approach in hematological malignancies and shows great promise in the MM field as well $(22,45,46)$. Nevertheless, in MM venetoclax use seems to only be beneficial for certain subgroup of patients and is detrimental for others, making it crucial to identify reliable biomarkers (47). Studies have highlighted the BCL2/MCL1 and BCL2/BCL2L1 expression ratios as predictors of response (48). However, in routine clinical practice these analyses are not performed and venetoclax use is confined to the $t(11 ; 14)$ setting, as these cases express high levels of BCL2 $(22,48)$. Since BCL2 over-expression seems to not be confined to $t(11 ; 14)$ cases, we mined our data to look for additional genomic predictors of response to venetoclax studying the expression levels of $B C L 2, M C L 1$, and $B C L 2 L 1$. Interestingly, as described in mouse models (49), $C Y L D$-deleted cases showed the most significant upregulation of $B C L 2$, while $\mathrm{t}(11 ; 14)$ cases did not cross our FDR threshold (Supplementary Table S2) for BCL2 expression unless associated with $\operatorname{del}(C Y L D)$. In $\mathrm{t}(11 ; 14)$, the most notable feature was a down-regulation of $B C L 2 L 1$. Conversely, chr(1q21)gain/amps alone or in association with $\operatorname{chr}(13)$ deletions were characterized by an overexpression of MCL1 (Supplementary Table S2 and Fig. 5), likely explaining resistance to venetoclax of $\operatorname{chr}(1 \mathrm{q})$ cases, who, by contrast, are highly sensitive to MCL-1 targeting (50). Indeed, among BCL2 over-expressing cases, there was an enrichment of $\mathrm{t}(11 ; 14)$ and $\operatorname{del}(C Y L D)$ (Supplementary Fig. S11A). MCL1 expression was characterized by an enrichment of chr(1q)gain/amps among high-expressors (Supplementary Fig. S11B). In the case of BCL2L1, it was clearly seen how most cases with low expression were indeed characterized by $t(11 ; 14)$ (Supplementary Fig. S11C). Concerning expression ratios, chr(1q)gain/amps were associated with a significant reduction of the $B C L 2 / M C L 1$ ratio. On the other hand, the $B C L 2 / B C L 2 L 1$ ratio was very high in the $\mathrm{t}(11 ; 14)$ setting, explaining the positive effect of venetoclax in this subgroup (Supplementary Table S3).

In conclusion, we observed genetic lesions that are associated with a peculiar modulation of expression of genes related to the BCL2 pathway which could translate into biomarkers for venetoclax treatment.

\section{In-silico drug sensitivity screening shows that $t(11 ; 14)$ is not the only predictor for Venetoclax response}

The above results highlighted some genotype-phenotype correlations that may predict response to BCL2 pathway inhibitors. To validate these findings in silico, we interrogated public datasets generated within the Cancer Dependency Map (DepMap) international collaboration, reporting on genetic dependencies and small molecule sensitivities of about 2000 cancer cell lines $(33,34)$. To select the most appropriate cell lines for our analysis, we used the Celligner algorithm (35). This allowed the comparison of transcriptomic data from DepMap cell lines and MM samples from CoMMpass, and therefore the selection of the cell lines most similar to our primary sample data. MM cells lines are characterized by specific IGH rearrangements, and Celligner correctly clustered them with MM samples harboring the same translocation (Fig. 6A). Interestingly, even if HD MM cell lines are hardly reported, many cell lines were anchored with HD patient samples, which may have translational relevance. Analysis of genomic and expression data showed that this was not due to $M Y C$ translocation or overexpression. In the sample space defined by Celligner we selected cell lines harboring the three chromosomal abnormalities that we identified above as possible predictors of venetoclax response (features of cell lines are summarized in Supplementary Table S4). The cell lines GRANTA519 and MM cell lines SKMM2 and KMS12BM harbor a t(11;14) translocation, and a $C Y L D$ deletion in the latters. As expected, these three cell lines resulted highly sensitive to Venetoclax treatment (Fig. 6B-D). Then, we interrogated the dataset for the U266 and MOLP8 MM cell lines, both carrying a $\mathrm{t}(11 ; 14)$. Notably, these two models showed refractoriness to BCL2 inhibition (Fig. 6E-F), most likely since they are both characterized by high expression of MCL1 due to chr(1q)gain/amp, and BCL2L1 gain in the case of U266. The only cell line in DepMap with 
an isolated del(CYLD) was the RI1, and it was sensitive to venetoclax (Fig. 6G). To gain further evidence that $\operatorname{del}(C Y L D)$ could predict venetoclax response, we analyzed public data on venetoclax sensitivity reported in recent papers (50-52). By integrating these drug sensitivity data and genomic information retrieved from literature and publicly available repositories, we were able to report the characteristics of 53 additional MM cell lines (Supplementary Table S5). Five of these harbored a $\operatorname{del}(C Y L D)$, but this was isolated only in PCM6 and in the remaining 4 lines was associated with chr(1q21)gain/amps. Adding independent evidence to our hypothesis, the PCM6 cell line resulted highly sensitive to venetoclax (51), while the others were resistant likely due to MCL1 overexpression. In fact, the MCL1 inhibitor S63845 was tested in the AMO1 cell line harboring a $\operatorname{chr}(1 \mathrm{q})$ amp and resulted in decreased viability (50).However, the complex genotype of cell lines and the many genetic and epigenetic variables affecting expression represent major challenges to the discovery and wider applicability of candidate biomarkers of venetoclax sensitivity, and validation by functional studies will clearly be required. Extensive mining of geno-transcriptomic data and cancer dependencies may increase the spectrum of candidate biomarkers of venetoclax sensitivity in MM.

\section{DISCUSSION}

In this study, we systematically dissected the impact of well-known and more recently described genome-wide DNA abnormalities on the expression profile of $514 \mathrm{MM}$ cases to identify putative functional correlates and surrogates of gene expression that may have translational implications. As expected, and confirming the validity of our approach, we found that hyperdiploidy and recurrent IGH translocations were associated with the most substantial differential gene expression patterns between patients. The assessment of the transcriptomic profiles associated with any recurrent CNA and gene mutation, representing a hallmark of our analysis, highlighted novel correlates, by means of which we could also identify specific lesions worthy of investigation in clinical-grade diagnostics for their potential implications on patient management. Overall, gene mutations carried a lower transcriptomic impact. This may be explained by the fact that most show instances of convergent evolution (e.g., mutually exclusive mutations of KRAS and NRAS), do not segregate with specific genomic lesions, and are often subclonal. Therefore, their impact may be diffuse and redundant, and furthermore diluted when analyzing bulk sequencing data. Interestingly though, functional relevance seems to be only attributable to hotspot mutations for the two most common mutated genes KRAS and NRAS, and their hotspot usage is quite different between MM and other solid cancers. This finding may have functional implications that needs to be further studied. A questionable driver event is represented by IGLL5 mutations, as they are mostly seen as off-target AID-induced mutations (53). The fact that in our analysis IGLL5 mutations are not associated with the differential expression of any transcript strongly supports this view. However, they could be a marker of IGL translocations and poor prognosis (2).

The single CNA associated with the largest number of transcriptomic correlates was $\operatorname{chr}(1 \mathrm{q} 21)$, where gains and, to a more extent, amplifications predicted downregulation of $P D-L 1$ and upregulation of SLAMF7, GPRC5D and MCL1, with clear implications for treatment with immunotherapies and BCL2 inhibitors. Overall, chr(1q21)gain/amp corresponded to a transcriptional modulation that was genome-wide and not limited to that genomic region, and was associated with the upregulation of a proliferative program driven by CCND2 and other oncogenes. Indeed, a similar pattern has been reported by RNAseq of proteasome inhibitors and immunomodulatory agents double-refractory cases (7), making chr(1q)gain/amp one of the worst prognostic markers even in the era of novel treatments (54). Of note, transcriptomic changes associated with chr(1q21) were much more profound in case of amplifications than gains. This was an overall very notable trend also for tumor suppressor lesions, where most of the transcriptomic changes were found associated with bi-allelic events (i.e., mutation of one allele and deletion of the other). Some of these had therapeutic correlates, such as NK-kB pathway upregulation in TRAF3 
and $C Y L D$ inactivation, predicting good response to PI treatment $(55,56)$. Others had prognostic value, such as PHF19 upregulation that was seen upon TP53 bi-allelic loss (41).

Most importantly, we identified potential genomic correlates of response to BCL2 pathway inhibitors. While response to venetoclax is best associated with a high BCL2/MCL1 or $B C L 2 / B C L 2 L 1$ ratio, this is rarely assessed in the clinic, and the easier-to-identify $t(11 ; 14)$ translocation is the typical biomarker used to select patients that may respond to venetoclax. Our data show how $\mathrm{t}(11 ; 14)$ patients display high $B C L 2$ levels and lack chr(1q21)gain/amp events thus showing low MCL1 levels. However, their sensitivity to venetoclax is best explained by their consistently low BCL2L1 levels. In our in silico analysis, $\mathrm{t}(11 ; 14)$ was found associated with resistance to venetoclax therapy in two cell lines, where it co-occurred with amplification of $1 \mathrm{q}$ and high expression of MCL1. In this setting, Slomp et al have shown how 1q amplifications could identify a high-risk patient subset suitable for therapy with specific MCL1 inhibitors. Interestingly, the authors have also shown how the combinatorial therapy with BCL2 and MCL1 antagonists could overcome venetoclax resistance in human myeloma cell lines as well as the newly diagnosed patients (50). Of note, the double inhibition leads to the formation of pro-apoptotic BAX-BAK hetero-complexes promoting apoptosis activation (52), and this could be the mechanism restoring apoptosis in these cells. Our systematic analysis also allowed to identify other DNA lesions that may predict venetoclax response, and among these are $C Y L D$ deletions. These are not significantly associated with $\mathrm{t}(11 ; 14)(10,14)$, and are associated with the highest BCL2 levels of the CoMMpass cohort. Consistently, our functional in silico validation confirmed that $\operatorname{del}(C Y L D)$ cell lines respond to venetoclax and that this could represent a candidate biomarker to identify patients who could respond to BCL2 inhibition. Nevertheless, within MM cell lines, CYLD deletions were more variably associated with venetoclax sensitivity, owing to complex karyotypes with additional chromosomal abnormalities involving BCL2 losses and chr(1q21)gain/amp associated with high MCL1 expression. Therefore, while our data from primary samples recapitulate in vivo mouse models where CYLD knock-down resulted in BCL2 upregulation (49), the translation of this evidence into a suitable biomarker of venetoclax sensitivity in the context of plasma cell neoplasms is hampered by the scanty cell line models with a clean genetic background, and likely by nongenetic mechanisms of resistance (57), and will require additional studies. In search for additional predictors of venetoclax sensitivity, Gupta et al. recently highlighted a new gene/antigen signature that is independent of $\mathrm{t}(11 ; 14)(51)$, and whose clinical utility will need testing.

Altogether, our study highlights the impact of detailed molecular profiling partnered with clinical annotations and how, when combined with large datasets of patients, this can be leveraged to study outcomes of targeted drugs in well-defined biological subgroups of MM patients.

\section{ACKNOWLEDGMENTS}

This work was supported by the European Research Council under the European Union's Horizon 2020 research and innovation program (817997 to N. Bolli); Associazione Italiana Ricerca sul Cancro (IG16722, IG10136 and IG24365 to A. Neri, IG20541); the Multiple Myeloma Research Foundation (MMRF) to F. Maura; the Perelman Family Foundation to O. Landgren; the Riney Family Multiple Myeloma Research Program Fund to O. Landgren; a Memorial Sloan Kettering Cancer Center NCI Core Grant (P30 CA 008748 to O. Landgren); and a Sylvester Comprehensive Cancer Center NCI Core Grant (P30 CA 240139 to O. Landgren).

F.Maura is supported by the American Society of Hematology.

\section{DISCLOSURE}

All authors declare that the research was conducted in the absence of any commercial or financial relationships that could be construed as a potential conflict of interest. N. Bolli received honoraria from Celgene, Amgen and Janssen. F. Iorio receives funding from Open Targets, a public-private 
initiative involving academia and industry and performs consultancy for the joint CRUKAstraZeneca Functional Genomics Center. 


\section{REFERENCES}

1. Morgan GJ, Walker BA, Davies FE. The genetic architecture of multiple myeloma. Nat Rev Cancer 2012;12(5):335-48 doi 10.1038/nrc3257.

2. D'Agostino M, Zaccaria GM, Ziccheddu B, Rustad EH, Genuardi E, Capra A, et al. Early Relapse Risk in Patients with Newly Diagnosed Multiple Myeloma Characterized by Nextgeneration Sequencing. Clin Cancer Res 2020;26(18):4832-41 doi 10.1158/10780432.CCR-20-0951.

3. Keats JJ, Chesi M, Egan JB, Garbitt VM, Palmer SE, Braggio E, et al. Clonal competition with alternating dominance in multiple myeloma. Blood 2012;120(5):1067-76 doi 10.1182/blood-2012-01-405985.

4. Samur MK, Aktas Samur A, Fulciniti M, Szalat R, Han T, Shammas M, et al. GenomeWide Somatic Alterations in Multiple Myeloma Reveal a Superior Outcome Group. J Clin Oncol 2020;38(27):3107-18 doi 10.1200/JCO.20.00461.

5. Da Via MC, Ziccheddu B, Maeda A, Bagnoli F, Perrone G, Bolli N. A Journey Through Myeloma Evolution: From the Normal Plasma Cell to Disease Complexity. Hemasphere 2020;4(6):e502 doi 10.1097/HS9.0000000000000502.

6. Bolli N, Maura F, Minvielle S, Gloznik D, Szalat R, Fullam A, et al. Genomic patterns of progression in smoldering multiple myeloma. Nat Commun 2018;9(1):3363 doi 10.1038/s41467-018-05058-y.

7. Ziccheddu B, Biancon G, Bagnoli F, De Philippis C, Maura F, Rustad EH, et al. Integrative analysis of the genomic and transcriptomic landscape of double-refractory multiple myeloma. Blood Adv 2020;4(5):830-44 doi 10.1182/bloodadvances.2019000779.

8. Oben B, Froyen G, Maclachlan KH, Leongamornlert D, Abascal F, Zheng-Lin B, et al. Whole-genome sequencing reveals progressive versus stable myeloma precursor conditions as two distinct entities. Nat Commun 2021;12(1):1861 doi 10.1038/s41467-021-22140-0.

9. Neuse CJ, Lomas OC, Schliemann C, Shen YJ, Manier S, Bustoros M, et al. Genome instability in multiple myeloma. Leukemia 2020;34(11):2887-97 doi 10.1038/s41375-0200921-y.

10. Bolli N, Biancon G, Moarii M, Gimondi S, Li Y, de Philippis C, et al. Analysis of the genomic landscape of multiple myeloma highlights novel prognostic markers and disease subgroups. Leukemia 2018;32(12):2604-16 doi 10.1038/s41375-018-0037-9.

11. Walker BA, Mavrommatis K, Wardell CP, Ashby TC, Bauer M, Davies F, et al. A highrisk, Double-Hit, group of newly diagnosed myeloma identified by genomic analysis. Leukemia 2019;33(1):159-70 doi 10.1038/s41375-018-0196-8.

12. Weinhold N, Ashby C, Rasche L, Chavan SS, Stein C, Stephens OW, et al. Clonal selection and double-hit events involving tumor suppressor genes underlie relapse in myeloma. Blood 2016;128(13):1735-44 doi 10.1182/blood-2016-06-723007.

13. Maura F, Bolli N, Angelopoulos N, Dawson KJ, Leongamornlert D, Martincorena I, et al. Genomic landscape and chronological reconstruction of driver events in multiple myeloma. Nat Commun 2019;10(1):3835 doi 10.1038/s41467-019-11680-1.

14. Walker BA, Mavrommatis K, Wardell CP, Ashby TC, Bauer M, Davies FE, et al. Identification of novel mutational drivers reveals oncogene dependencies in multiple myeloma. Blood 2018;132(6):587-97 doi 10.1182/blood-2018-03-840132.

15. Hideshima T, Bergsagel PL, Kuehl WM, Anderson KC. Advances in biology of multiple myeloma: clinical applications. Blood 2004;104(3):607-18 doi 10.1182/blood-2004-010037.

16. Mattioli M, Agnelli L, Fabris S, Baldini L, Morabito F, Bicciato S, et al. Gene expression profiling of plasma cell dyscrasias reveals molecular patterns associated with distinct IGH translocations in multiple myeloma. Oncogene 2005;24(15):2461-73 doi 10.1038/sj.onc.1208447. 
17. Zhan F, Huang Y, Colla S, Stewart JP, Hanamura I, Gupta S, et al. The molecular classification of multiple myeloma. Blood 2006;108(6):2020-8 doi 10.1182/blood-2005-11013458.

18. Cleynen A, Szalat R, Kemal Samur M, Robiou du Pont S, Buisson L, Boyle E, et al. Expressed fusion gene landscape and its impact in multiple myeloma. Nat Commun 2017;8(1):1893 doi 10.1038/s41467-017-00638-w.

19. Foltz SM, Gao Q, Yoon CJ, Sun H, Yao L, Li Y, et al. Evolution and structure of clinically relevant gene fusions in multiple myeloma. Nat Commun 2020;11(1):2666 doi 10.1038/s41467-020-16434-y.

20. Lagana A, Perumal D, Melnekoff D, Readhead B, Kidd BA, Leshchenko V, et al. Integrative network analysis identifies novel drivers of pathogenesis and progression in newly diagnosed multiple myeloma. Leukemia 2018;32(1):120-30 doi 10.1038/leu.2017.197.

21. Rashid NU, Sperling AS, Bolli N, Wedge DC, Van Loo P, Tai YT, et al. Differential and limited expression of mutant alleles in multiple myeloma. Blood 2014;124(20):3110-7 doi 10.1182/blood-2014-04-569327.

22. Kumar S, Kaufman JL, Gasparetto C, Mikhael J, Vij R, Pegourie B, et al. Efficacy of venetoclax as targeted therapy for relapsed/refractory $\mathrm{t}(11 ; 14)$ multiple myeloma. Blood 2017;130(22):2401-9 doi 10.1182/blood-2017-06-788786.

23. Bolli N, Genuardi E, Ziccheddu B, Martello M, Oliva S, Terragna C. Next-Generation Sequencing for Clinical Management of Multiple Myeloma: Ready for Prime Time? Front Oncol 2020;10:189 doi 10.3389/fonc.2020.00189.

24. Bolli N, Li Y, Sathiaseelan V, Raine K, Jones D, Ganly P, et al. A DNA target-enrichment approach to detect mutations, copy number changes and immunoglobulin translocations in multiple myeloma. Blood Cancer J 2016;6(9):e467 doi 10.1038/bcj.2016.72.

25. Yellapantula V, Hultcrantz M, Rustad EH, Wasserman E, Londono D, Cimera R, et al. Comprehensive detection of recurring genomic abnormalities: a targeted sequencing approach for multiple myeloma. Blood Cancer J 2019;9(12):101 doi 10.1038/s41408-0190264-y.

26. Ritchie ME, Phipson B, Wu D, Hu Y, Law CW, Shi W, et al. limma powers differential expression analyses for RNA-sequencing and microarray studies. Nucleic Acids Res 2015;43(7):e47 doi 10.1093/nar/gkv007.

27. Law CW, Chen Y, Shi W, Smyth GK. voom: Precision weights unlock linear model analysis tools for RNA-seq read counts. Genome Biol 2014;15(2):R29 doi 10.1186/gb-201415-2-r29.

28. Robinson MD, Oshlack A. A scaling normalization method for differential expression analysis of RNA-seq data. Genome Biol 2010;11(3):R25 doi 10.1186/gb-2010-11-3-r25.

29. Gerstung M, Pellagatti A, Malcovati L, Giagounidis A, Porta MG, Jadersten M, et al. Combining gene mutation with gene expression data improves outcome prediction in myelodysplastic syndromes. Nat Commun 2015;6:5901 doi 10.1038/ncomms6901.

30. Chang MT, Asthana S, Gao SP, Lee BH, Chapman JS, Kandoth C, et al. Identifying recurrent mutations in cancer reveals widespread lineage diversity and mutational specificity. Nat Biotechnol 2016;34(2):155-63 doi 10.1038/nbt.3391.

31. Yu G, Wang LG, Han Y, He QY. clusterProfiler: an R package for comparing biological themes among gene clusters. OMICS 2012;16(5):284-7 doi 10.1089/omi.2011.0118.

32. Subramanian A, Tamayo P, Mootha VK, Mukherjee S, Ebert BL, Gillette MA, et al. Gene set enrichment analysis: a knowledge-based approach for interpreting genome-wide expression profiles. Proc Natl Acad Sci $U$ S A 2005;102(43):15545-50 doi 10.1073/pnas.0506580102. 
33. Iorio F, Knijnenburg TA, Vis DJ, Bignell GR, Menden MP, Schubert M, et al. A Landscape of Pharmacogenomic Interactions in Cancer. Cell 2016;166(3):740-54 doi 10.1016/j.cell.2016.06.017.

34. Tsherniak A, Vazquez F, Montgomery PG, Weir BA, Kryukov G, Cowley GS, et al. Defining a Cancer Dependency Map. Cell 2017;170(3):564-76 e16 doi 10.1016/j.cell.2017.06.010.

35. Warren A, Chen Y, Jones A, Shibue T, Hahn WC, Boehm JS, et al. Global computational alignment of tumor and cell line transcriptional profiles. Nat Commun 2021;12(1):22 doi 10.1038/s41467-020-20294-x.

36. Agnelli L, Bicciato S, Mattioli M, Fabris S, Intini D, Verdelli D, et al. Molecular classification of multiple myeloma: a distinct transcriptional profile characterizes patients expressing CCND1 and negative for 14q32 translocations. J Clin Oncol 2005;23(29):7296306 doi 10.1200/JCO.2005.01.3870.

37. Zhou Y, Zhang Q, Stephens O, Heuck CJ, Tian E, Sawyer JR, et al. Prediction of cytogenetic abnormalities with gene expression profiles. Blood 2012;119(21):e148-50 doi 10.1182/blood-2011-10-388702.

38. Lionetti M, Barbieri M, Todoerti K, Agnelli L, Fabris S, Tonon G, et al. A compendium of DIS3 mutations and associated transcriptional signatures in plasma cell dyscrasias. Oncotarget 2015;6(28):26129-41 doi 10.18632/oncotarget.4674.

39. Munawar U, Rasche L, Muller N, Vogt C, Da-Via M, Haertle L, et al. Hierarchy of monoand biallelic TP53 alterations in multiple myeloma cell fitness. Blood 2019;134(10):836-40 doi 10.1182/blood.2019000080.

40. Solimando AG, Da Via MC, Leone P, Borrelli P, Croci GA, Tabares P, et al. Halting the vicious cycle within the multiple myeloma ecosystem: blocking JAM-A on bone marrow endothelial cells restores angiogenic homeostasis and suppresses tumor progression. Haematologica 2021;106(7):1943-56 doi 10.3324/haematol.2019.239913.

41. Ren Z, Ahn JH, Liu H, Tsai YH, Bhanu NV, Koss B, et al. PHF19 promotes multiple myeloma tumorigenicity through PRC2 activation and broad H3K27me3 domain formation. Blood 2019;134(14):1176-89 doi 10.1182/blood.2019000578.

42. Nijhof IS, Casneuf T, van Velzen J, van Kessel B, Axel AE, Syed K, et al. CD38 expression and complement inhibitors affect response and resistance to daratumumab therapy in myeloma. Blood 2016;128(7):959-70 doi 10.1182/blood-2016-03-703439.

43. Bjorklund CC, Baladandayuthapani V, Lin HY, Jones RJ, Kuiatse I, Wang H, et al. Evidence of a role for CD44 and cell adhesion in mediating resistance to lenalidomide in multiple myeloma: therapeutic implications. Leukemia 2014;28(2):373-83 doi 10.1038/leu.2013.174.

44. Bergsagel PL, Kuehl WM, Zhan F, Sawyer J, Barlogie B, Shaughnessy J, Jr. Cyclin D dysregulation: an early and unifying pathogenic event in multiple myeloma. Blood 2005;106(1):296-303 doi 10.1182/blood-2005-01-0034.

45. DiNardo CD, Jonas BA, Pullarkat V, Thirman MJ, Garcia JS, Wei AH, et al. Azacitidine and Venetoclax in Previously Untreated Acute Myeloid Leukemia. $N$ Engl J Med 2020;383(7):617-29 doi 10.1056/NEJMoa2012971.

46. Roberts AW, Davids MS, Pagel JM, Kahl BS, Puvvada SD, Gerecitano JF, et al. Targeting BCL2 with Venetoclax in Relapsed Chronic Lymphocytic Leukemia. $N$ Engl J Med 2016;374(4):311-22 doi 10.1056/NEJMoa1513257.

47. Kumar S, Harrison SJ, Cavo C, De La Rubia J, Popat R, Gasparetto C, et al. Updated results from BELLINI, a phase III study of venetoclax or placebo in combination with bortezomib and dexamethasone in relapsed/refractory multiple myeloma. American Society of Clinical Oncology Annual Meeting 2020. Volume 38. Virtual: J Clin Oncol; 2020.

48. Touzeau C, Maciag P, Amiot M, Moreau P. Targeting Bcl-2 for the treatment of multiple myeloma. Leukemia 2018;32(9):1899-907 doi 10.1038/s41375-018-0223-9. 
49. Hovelmeyer N, Wunderlich FT, Massoumi R, Jakobsen CG, Song J, Worns MA, et al. Regulation of B cell homeostasis and activation by the tumor suppressor gene CYLD. $J$ Exp Med 2007;204(11):2615-27 doi 10.1084/jem.20070318.

50. Slomp A, Moesbergen LM, Gong JN, Cuenca M, von dem Borne PA, Sonneveld P, et al. Multiple myeloma with 1q21 amplification is highly sensitive to MCL-1 targeting. Blood Adv 2019;3(24):4202-14 doi 10.1182/bloodadvances.2019000702.

51. Gupta VA, Barwick BG, Matulis SM, Shirasaki R, Jaye DL, Keats JJ, et al. Venetoclax sensitivity in multiple myeloma is associated with B-cell gene expression. Blood 2021;137(26):3604-15 doi 10.1182/blood.2020007899.

52. Seiller C, Maiga S, Touzeau C, Bellanger C, Kervoelen C, Descamps G, et al. Dual targeting of BCL2 and MCL1 rescues myeloma cells resistant to BCL2 and MCL1 inhibitors associated with the formation of BAX/BAK hetero-complexes. Cell Death Dis 2020;11(5):316 doi 10.1038/s41419-020-2505-1.

53. Maura F, Rustad EH, Yellapantula V, Luksza M, Hoyos D, Maclachlan KH, et al. Role of AID in the temporal pattern of acquisition of driver mutations in multiple myeloma. Leukemia 2020;34(5):1476-80 doi 10.1038/s41375-019-0689-0.

54. Abdallah N, Greipp P, Kapoor P, Gertz MA, Dispenzieri A, Baughn LB, et al. Clinical characteristics and treatment outcomes of newly diagnosed multiple myeloma with chromosome 1q abnormalities. Blood Adv 2020;4(15):3509-19 doi 10.1182/bloodadvances.2020002218.

55. Annunziata CM, Davis RE, Demchenko Y, Bellamy W, Gabrea A, Zhan F, et al. Frequent engagement of the classical and alternative NF-kappaB pathways by diverse genetic abnormalities in multiple myeloma. Cancer Cell 2007;12(2):115-30 doi 10.1016/j.ccr.2007.07.004.

56. Keats JJ, Fonseca R, Chesi M, Schop R, Baker A, Chng WJ, et al. Promiscuous mutations activate the noncanonical NF-kappaB pathway in multiple myeloma. Cancer Cell 2007;12(2):131-44 doi 10.1016/j.ccr.2007.07.003.

57. Neri P, Maity R, Alberge J, Sinha S, Donovan J, Kong M, et al. Mutations and Copy Number Gains of the BCL2 Family Members Mediate Resistance to Venetoclax in Multiple Myeloma (MM) Patients. In: Blood, editor. American Society of Hematology Annual meeting. San Diego, CA, USA2019. 


\section{FIGURE LEGENDS}

\section{Figure 1}

Transcriptomic profile. a) Principal component analysis based on the main karyotypic subtypes: samples are represented as dots in the space identified by the 3 principal components and are colorcoded based on their karyotype. b) Stacked bar chart showing the differential expression genes per each genomic abnormality (FDR $<0.05$, Fold Change cut-off 1.5). Bars indicate the contributions of up-regulated genes (red) and down-regulated genes (blue). c) Scatterplot representing expression prediction for the $C C N D 1$ and $C Y L D$ genes versus observed expression values: samples are colorcoded dots based on the significant genomic abnormality (FDR $<0.01)$, in blue $t(11 ; 14)$ samples, in green del $(C Y L D)$ samples and in black the other samples; $\mathrm{R}^{2}$ represents the association between genomic alteration and expression changes.

\section{Figure 2}

Hotspot versus nonHS and WT analysis. a-d) Lollipop plots for the 4 main mutated genes; each plot shows in the upper side the hotspots in MM, at the bottom the hotspots in the peculiar solid cancers. Lollipop-plots have been performed applying the R package "maftools. ai-di) Venn plots for each differential expression analysis.

\section{Figure 3}

Bi-Allelic Inactivation analysis. a-b) CYLD and TP53 analysis. In the left upper panel, the z-score value heatmaps of the cumulative effect genes, where columns represent the gene status and rows represent the genes. In the right upper panel, the Gene-Network plot for cumulative effect genes, where big dots indicate the enriched pathways, small dots represent the deregulated genes, and the color-coding refers to the fold-change in bi-allelic versus WT contrast $(\log 2 \mathrm{CPM})$. In the lower panel the box plots represent the cumulative gene expression trend in the 3 statuses, for each analysis: the dots specify the samples in each status, the red line symbolizes the trend of the gene expression, the red number denotes the incremental or decremental expression fold-change, the black number the p-value.

\section{Figure 4}

Chromosome 1q gain/amplification analysis. a) The heatmap represents the z-score values for the cumulative effect genes. b) The Pie chart represents the chromosomal distribution of the cumulative effect genes, corrected for chromosomal length. c) Gene-Network plot for cumulative effect genes, related to the amplification versus WT contrast $(\log 2 \mathrm{CPM})$. d) Box plot for the SLAMF7 expression trend in the 3 statuses. e) Box plot for $M C L 1$ expression trend.

\section{Figure 5}

BCL2 family gene expression analysis. Box plots representing the specific BCL2 family gene expression in $\mathrm{t}(11 ; 14), \operatorname{del}(C Y L D)$ and gain-amp(1q) samples.

\section{Figure 6}

In-silico drug sensitivity screen. a) UMAP 2D projection of Celligner-aligned sample and cell line expression data: dots represent the samples (HD in light blue, $t(4 ; 14)$ in yellow, $t(11 ; 14)$ in blue, $\mathrm{t}(14 ; 16)$ in salmon and $\mathrm{t}(14 ; 20)$ in pink) and the lymphoma (dark blue) and myeloma (red) cell lines, samples missing karyotype in grey. b-g) Dose-Response curves for cell lines of interest: where orange dots represent tests and blue curves represent the fitted trends. $d r c, d r 4 p l \mathrm{R}$ packages have been used to perform drug-sensitivity plots. 


\section{KRAS}

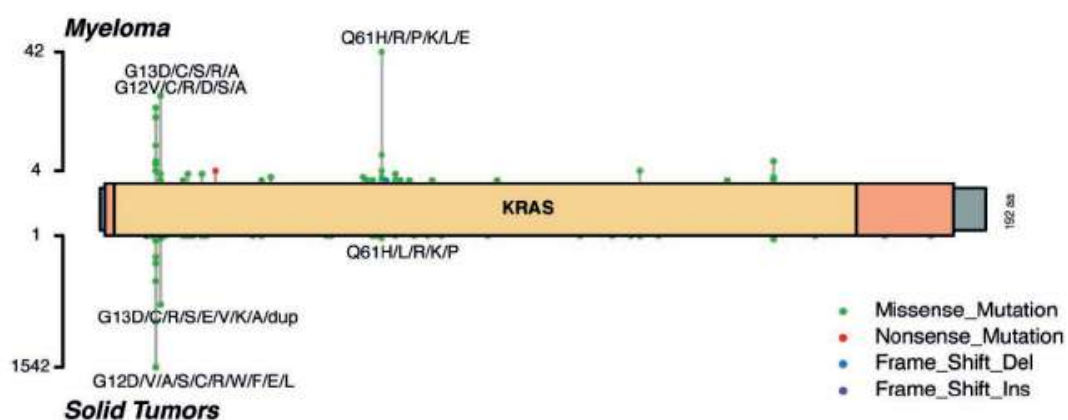

\section{NRAS}

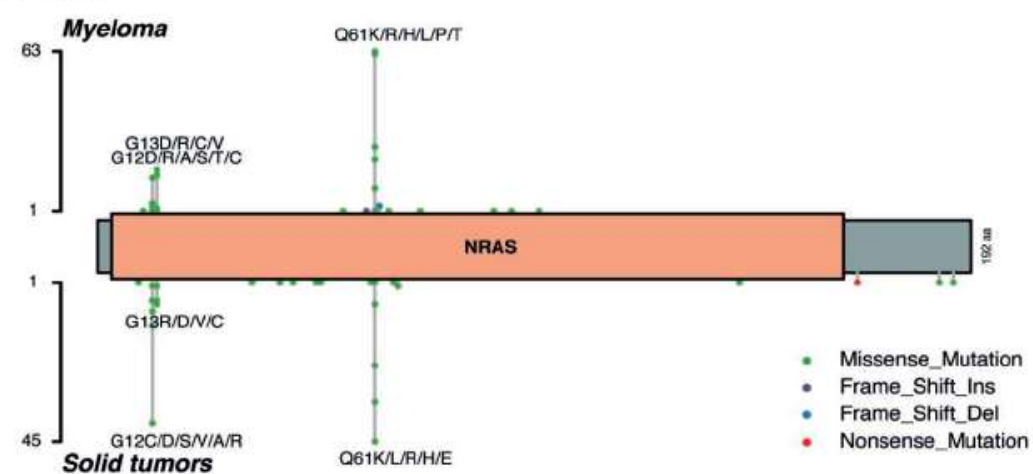

\section{C}

\section{IRF4}

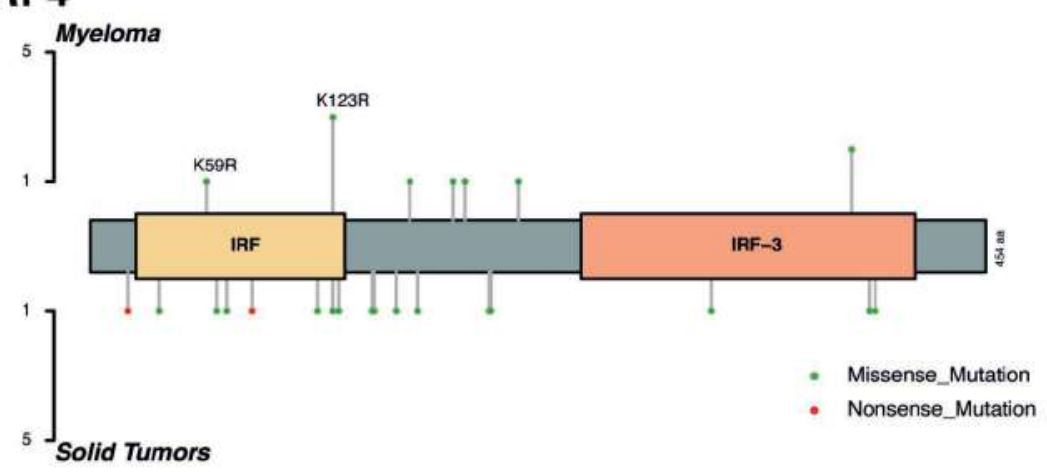

\section{BRAF}

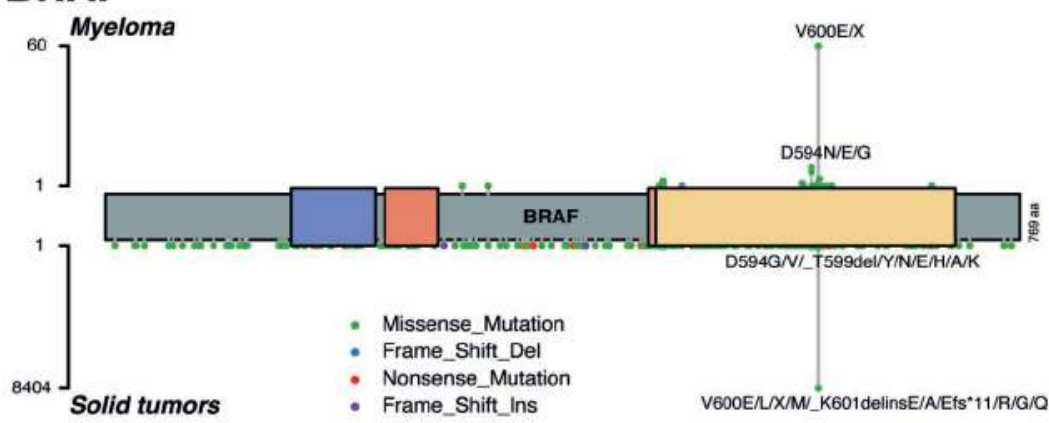

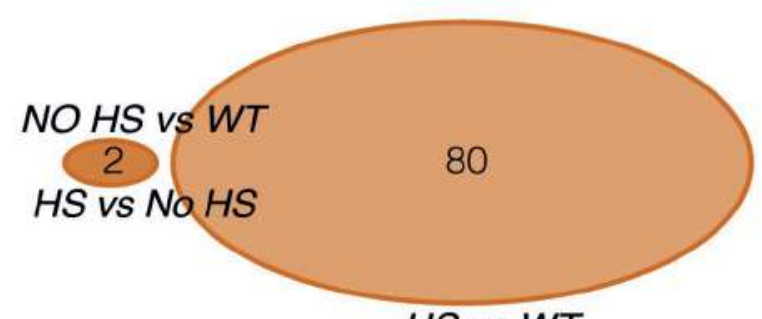

HS vs WT

bi

ci

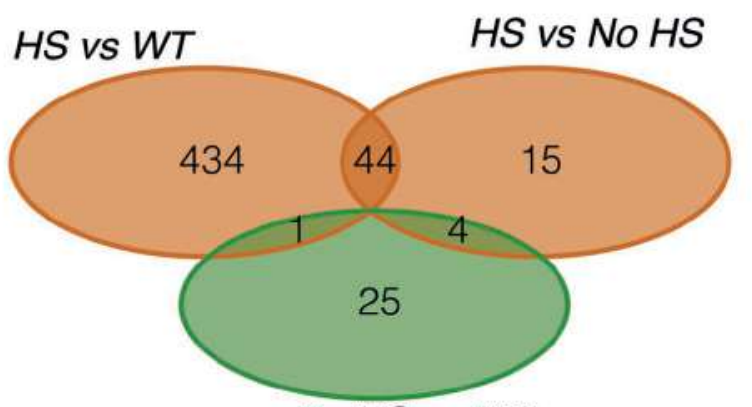

No HS vs WT
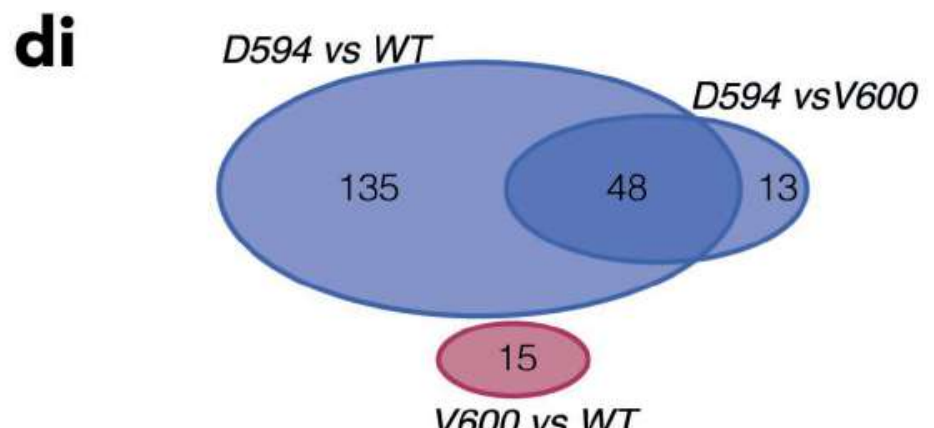

DEGs in HotSpot

DEGs in NO-HotSpot

DEGs in D594

\section{Figure 2}



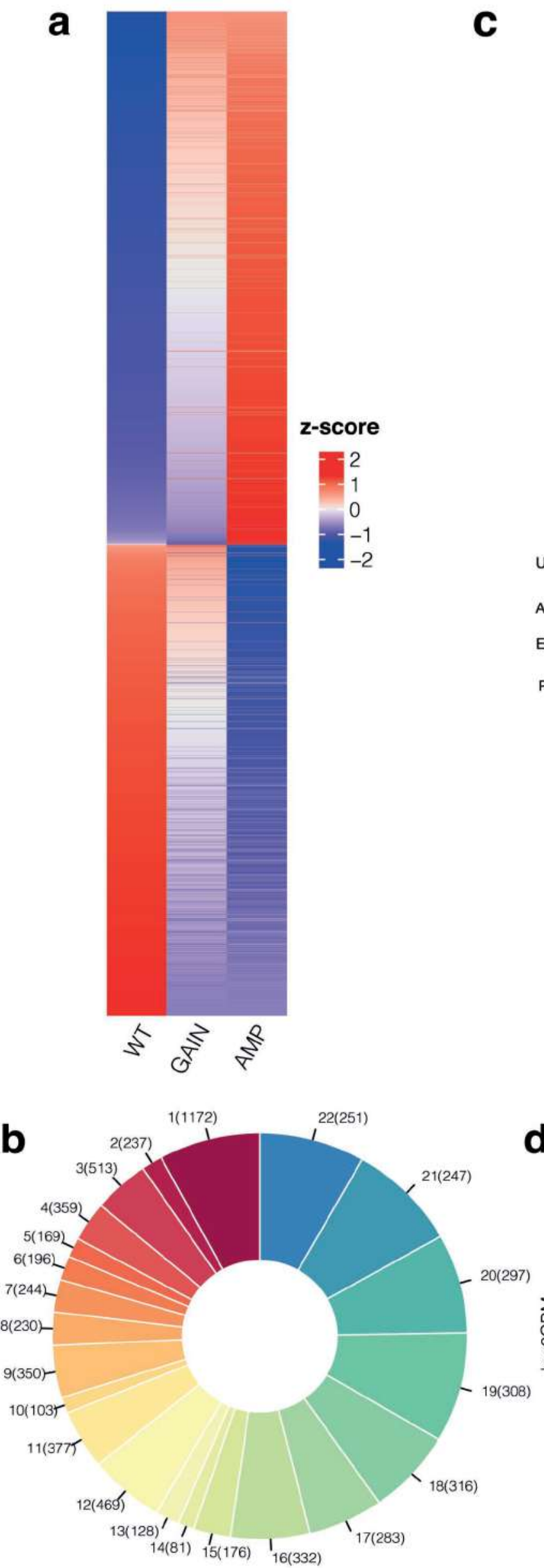

Figure 4

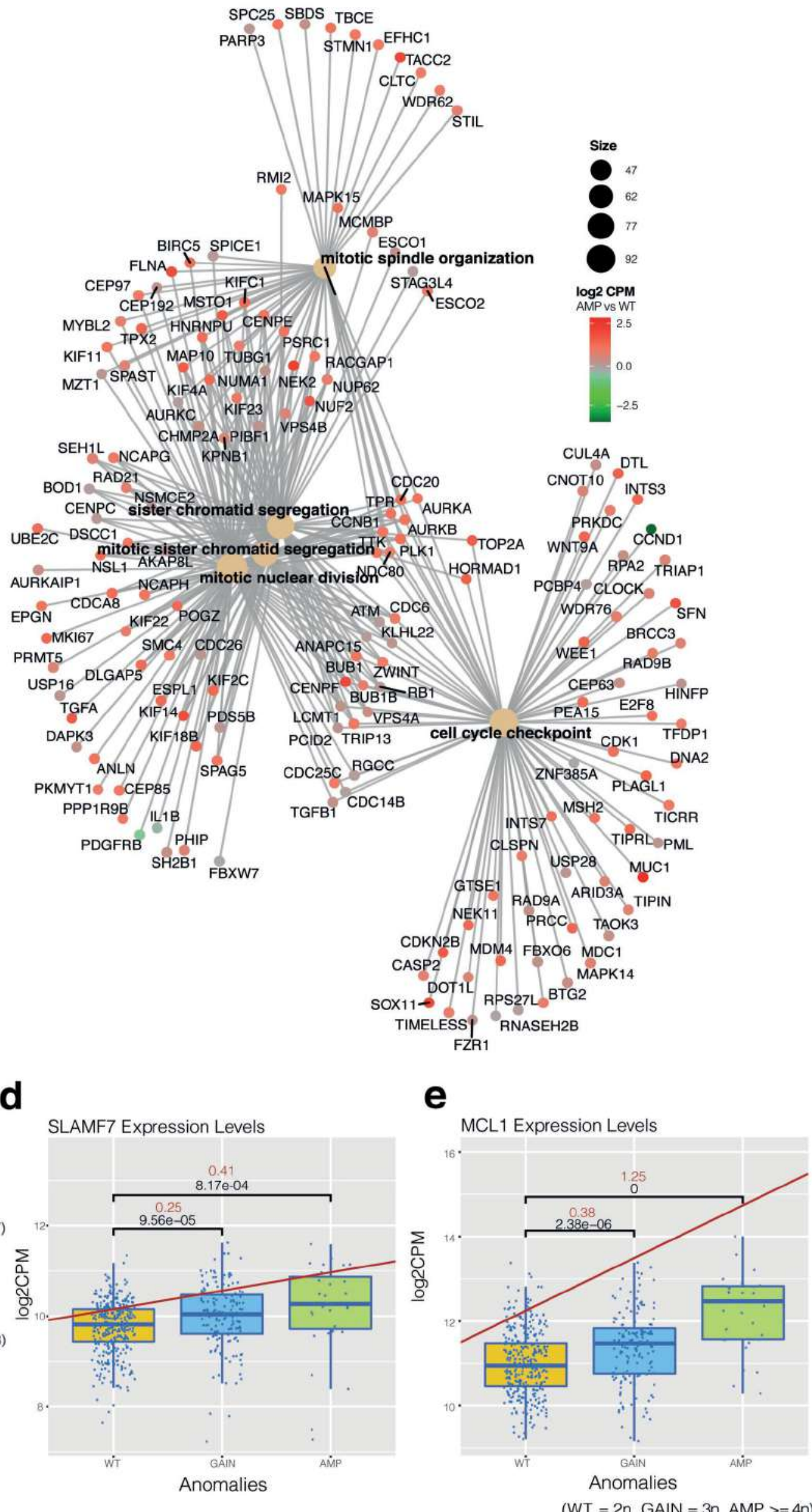



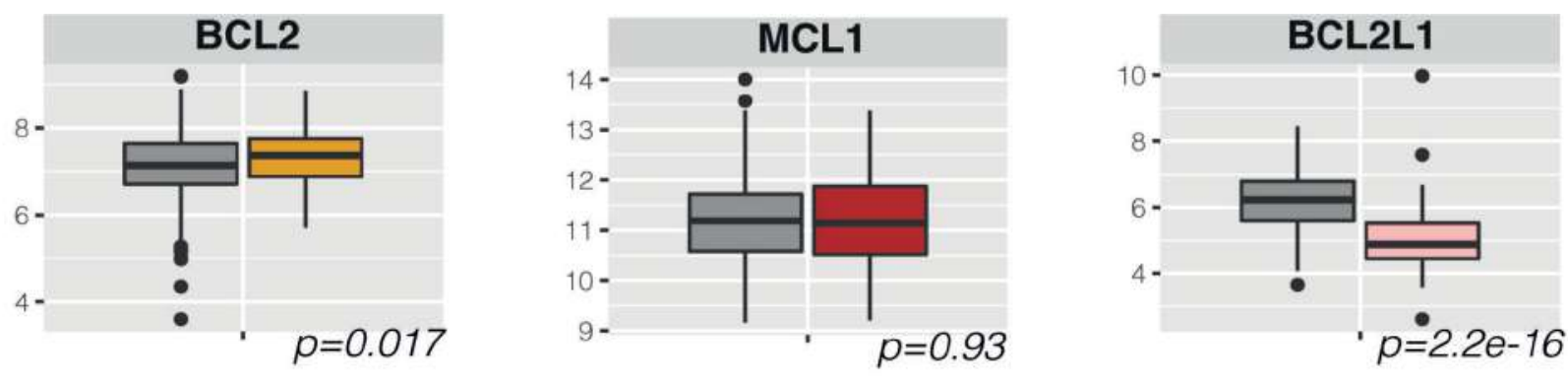

t.11.14
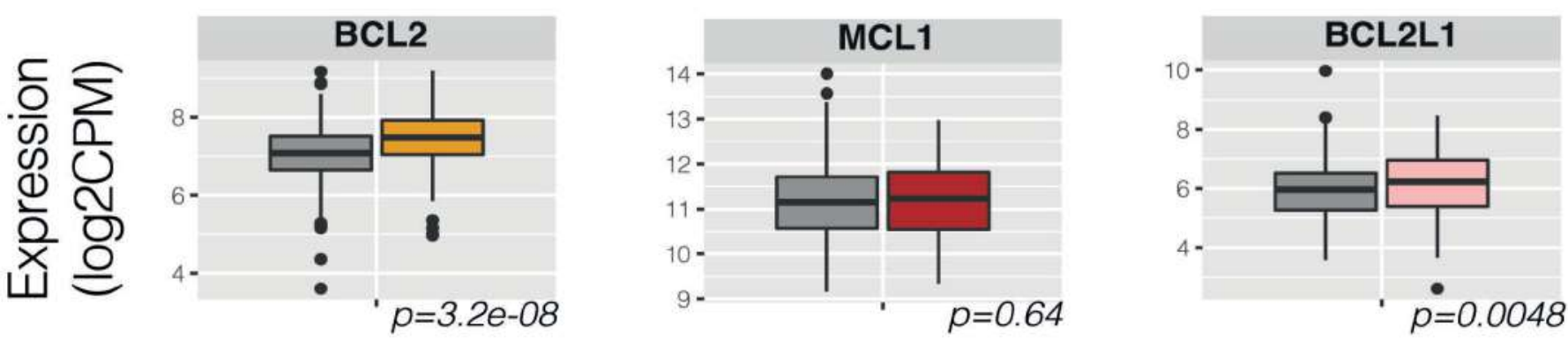

delCYLD
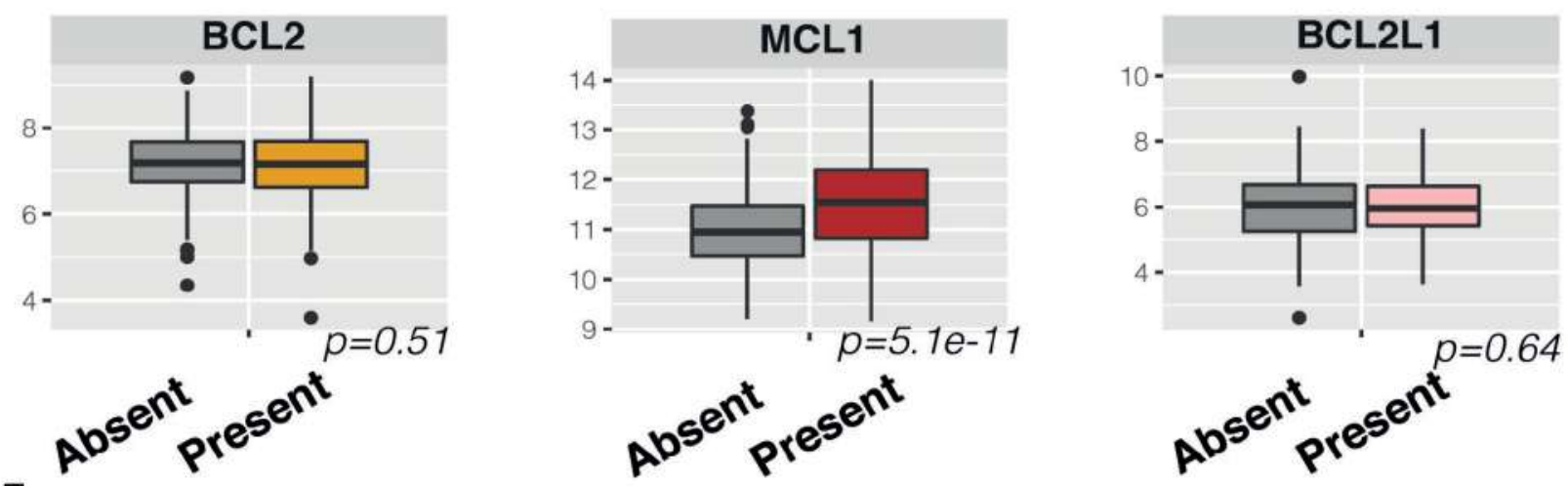

Gain-Amp1q

Figure 5

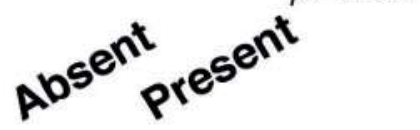


a

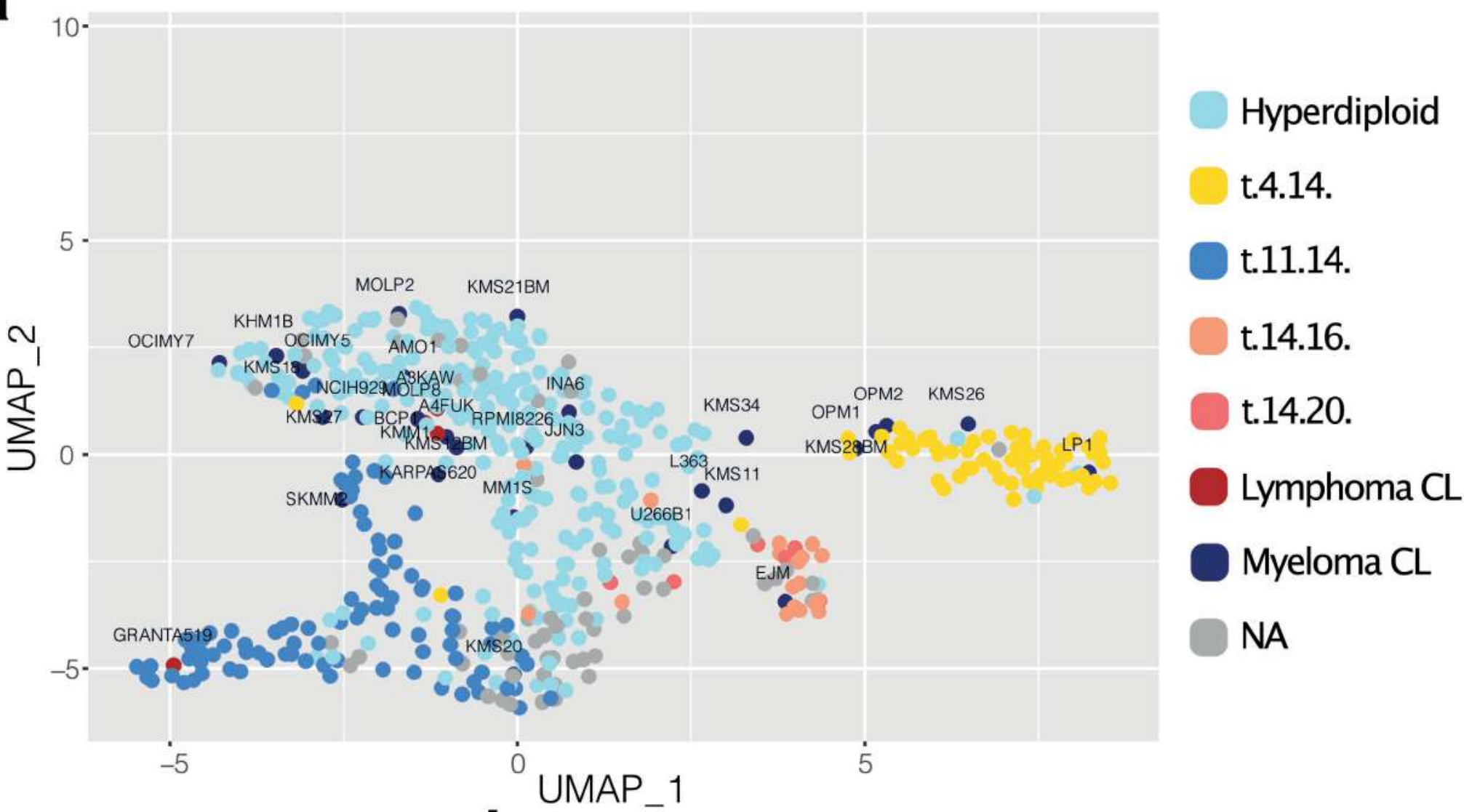

b

GRANTA519 - Venetoclax

t.11.14

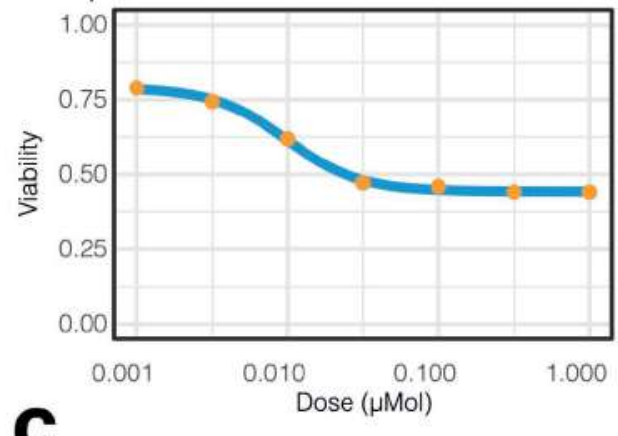

SKMM2 - Venetoclax

t.11.14, delCYLD

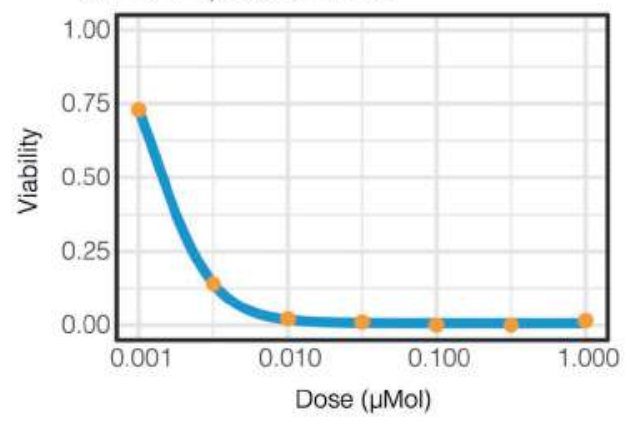

Figure 6 d

KMS12BM - Venetoclax t.11.14, delCYLD

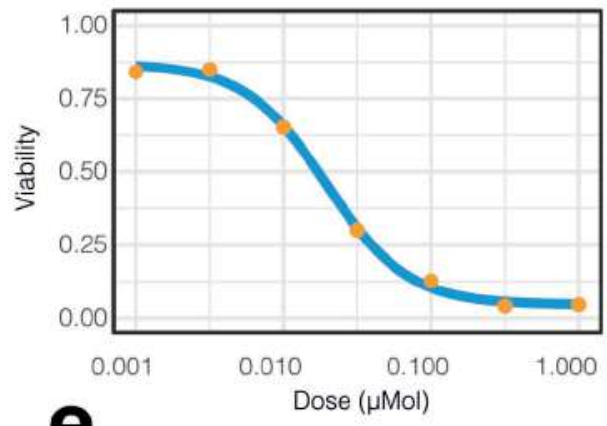

MOLP8 - Venetoclax

t.11.14 \& amp1q21

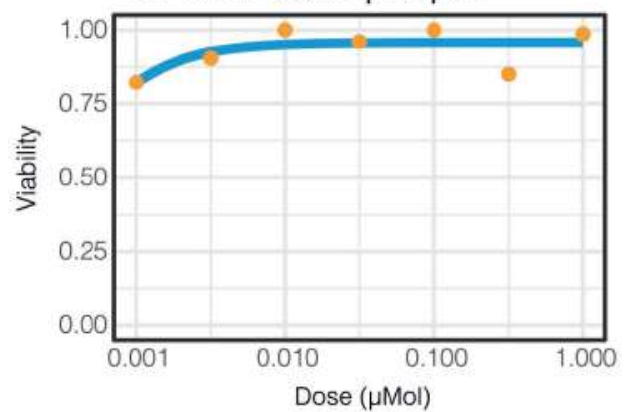

Curve

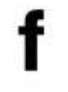

U266 - Venetoclax

t.11.14 \& gain1q21

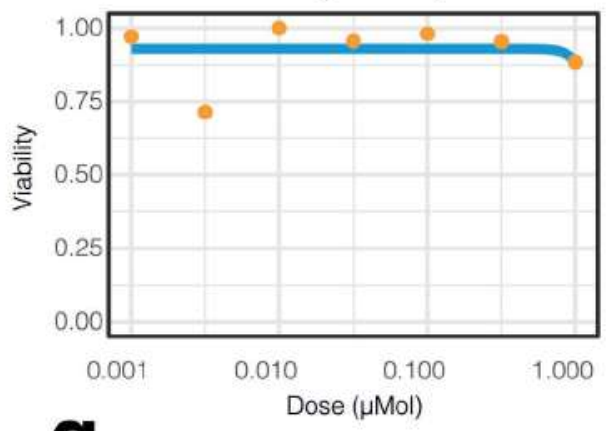

9 RI1 - Venetoclax delCYLD

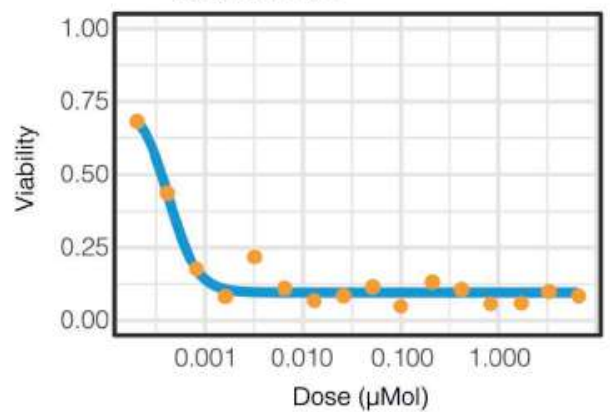




\section{Functional impact of genomic complexity on the transcriptome of Multiple Myeloma}

Bachisio Ziccheddu, Matteo Claudio Da Via, Marta Lionetti, et al.

Clin Cancer Res Published OnlineFirst September 15, 2021.

\section{Updated version Access the most recent version of this article at: doi:10.1158/1078-0432.CCR-20-4366}

Supplementary Access the most recent supplemental material at:

Material http://clincancerres.aacrjournals.org/content/suppl/2021/09/14/1078-0432.CCR-20-4366.DC1

Author Author manuscripts have been peer reviewed and accepted for publication but have not yet Manuscript been edited.

E-mail alerts Sign up to receive free email-alerts related to this article or journal.

Reprints and To order reprints of this article or to subscribe to the journal, contact the AACR Publications Subscriptions Department at pubs@aacr.org.

Permissions To request permission to re-use all or part of this article, use this link http://clincancerres.aacrjournals.org/content/early/2021/09/14/1078-0432.CCR-20-4366. Click on "Request Permissions" which will take you to the Copyright Clearance Center's (CCC) Rightslink site. 